\title{
In situ Imaging of Materials using X-ray Tomography
}

\author{
Brian M. Patterson ${ }^{1}$, Nikolaus L. Cordes ${ }^{1}$, Kevin Henderson ${ }^{1}$, Xianghui Xiao², and Nikhilesh Chawla ${ }^{3}$ \\ 1. Materials Science and Technology Division, Engineered Materials Group, Los Alamos National \\ Laboratory, Los Alamos, NM, USA \\ 2. X-ray Photon Sciences, Argonne National Laboratory, Argonne, IL, USA \\ 3. 4D Materials Science Center, Arizona State University, Tempe, AZ
}

X-ray computed tomography (X-ray CT) has become an indispensable tool in the understanding of material morphology. X-ray CT can provide a full 3D, nondestructive image of a material to understand the internal structure (e.g., voids, cracks, phase distribution, or even particle size and shape) which can then be measured. Since the technique is nondestructive, it is possible to collect images of materials before, and after other ancillary experiments are completed. These experiments may include: loading (compressive or tensile), thermal[1] (heating or cooling), electrical (cyclical testing), biological (growth rates), or chemical [2] (corrosion changes). Nowhere is this technique more powerful than when the experiment is conducted in situ. For example, imaging the bending, buckling, breaking, and fracture propagation of a foam, in real time, while a load is placed on the material and the X-ray CT images are collected simultaneously. However, in situ X-ray CT has many challenges in order to make the experiment meaningful.

Several types of in situ experiments may be completed. These include: pre- and post- experiment, interval in situ, interrupted in situ [3, 4], and finally dynamic in situ [5, 6]. Each of these types of experiments has their own challenges and advantages. X-ray CT begins as a series of X-ray radiographs that are collected as the material is rotated $180^{\circ}$. These radiographs are then reconstructed and rendered. The speed at which the radiographs need to be, or can be collected, may determine the type of experiment. Additionally, upon collecting the radiographs, post processing the data to obtain the material science information needed, may be a long and complicated series of steps, Figure 1. Each of these steps may be completed using a different software packages from the previous step, and on several terrabytes of data. Automation is critical.

This presentation will outline the challenges in obtaining in situ X-ray CT data and the processing steps (Figure 2) to take the reconstructed image to a rendered and quantifiable data set. This is followed by a multitude of post processing opportunities such as correlating the images to the in situ information (e.g. temperature, load, etc.), modeling the data, using other advanced processing techniques such as digital volume correlation data and relating it all to the in situ images (Figure 3). Finally, advanced statistical processing techniques such as relating the morphological measures (e.g. equivalent diameter, sphericity, percent void volume) of objects within the image to their changes during the experiment are required to relate how those measures connect the structure-property relationships.

References:

[1] N.C. Chapman, et. al., Materials Science and Technology 31(5) (2015) 573-578.

[2] S.S. Singh, et. al., Corrosion Science 104 (2016) 330-335.

[3] B.M. Patterson, et. al., Exp Mech 56(9) (2016) 1585-1597.

[4] B.M. Patterson, et. al., Microscopy and Microanalysis 95 (2014) 18-26.

[5] J.C.E. Mertens, et. al., Journal of Materials Science 52(20) (2017) 12185-12206.

[6] B.M. Patterson, et. al., Journal of Material Science 51(1) (2016) 171-187. 


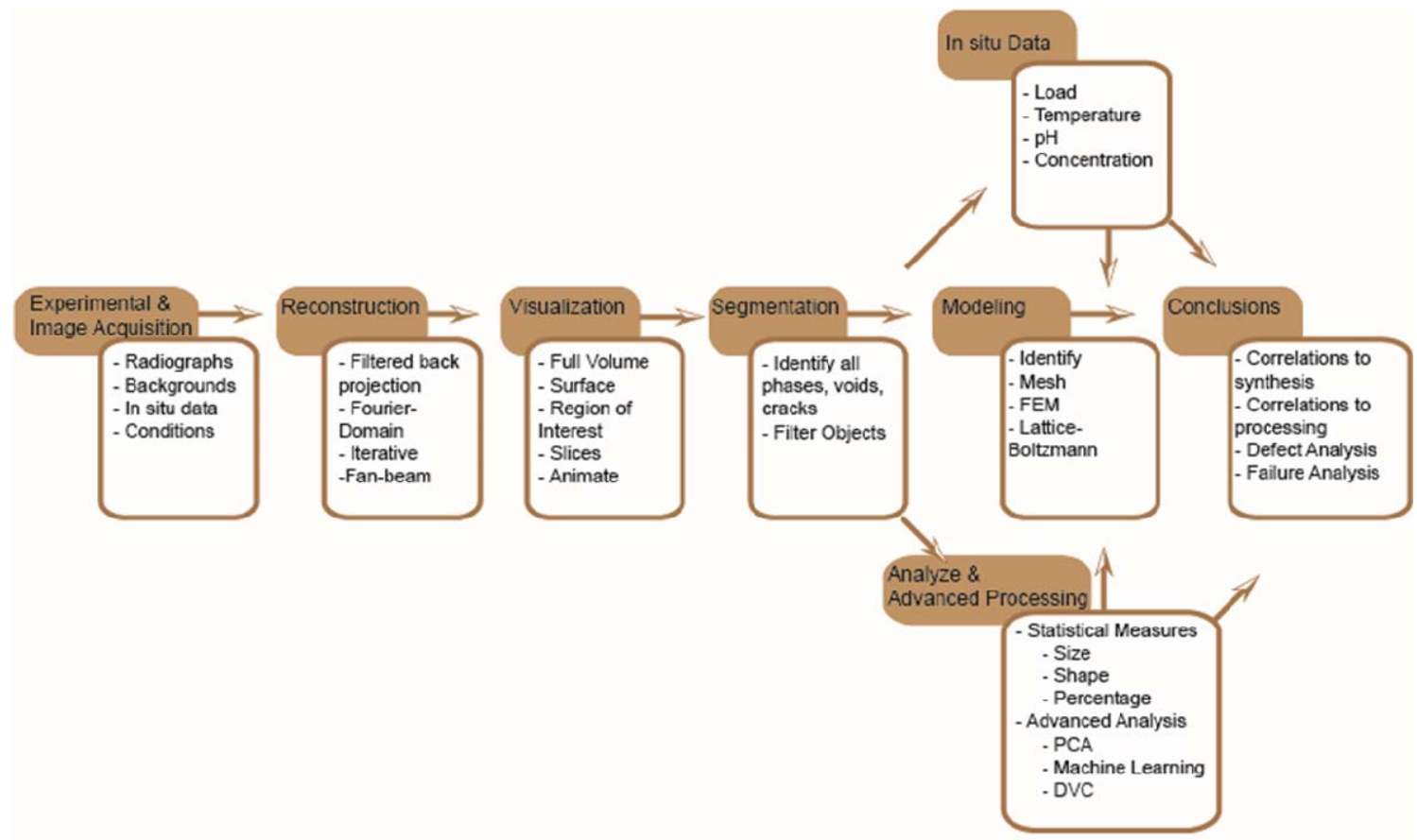

Figure 1. Outline of the multitude of steps required to process the data of an in situ X-ray CT experiment. Each step may require a different software package and automation is required when processing several terrabytes of data.

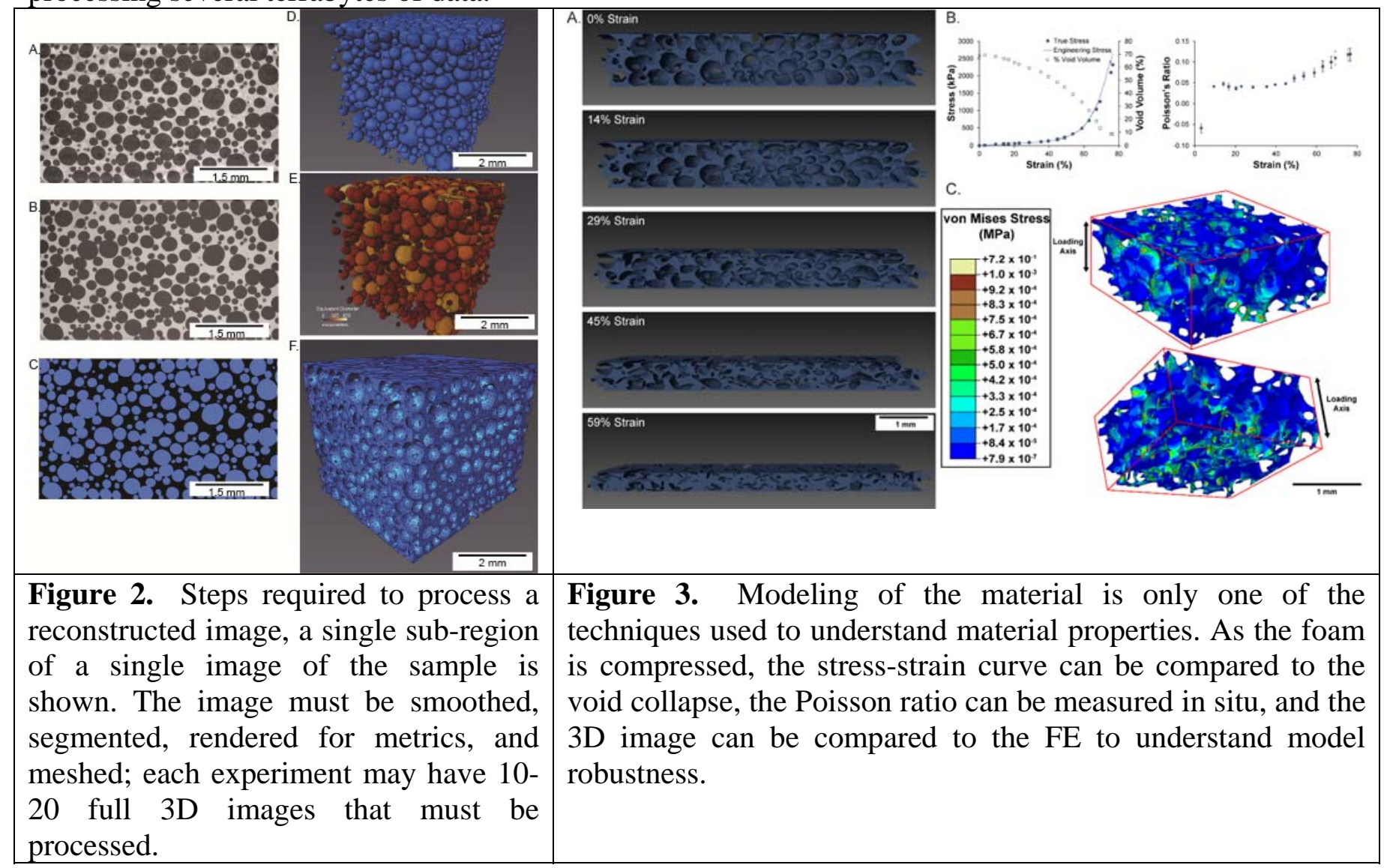

